\title{
Teacher School Mental Health Literacy Survey: A Validation Study
}

\author{
Courtney R. Mastrorio, Kathryn M. Trainor, Joni W. Splett \\ University of Florida
}

Faculty mentor: Joni W. Splett, College of Education

\begin{abstract}
Since teachers play an instrumental role in identifying students with social-emotional or behavioral (SEB) concerns, there is a need to assess and improve teachers' knowledge in relation to children's mental health. This study examines the psychometric properties of one such measure, Teacher School Mental Health Literacy Survey (TSMHLS), within a sample of pre-service teachers $(n=38$.) The survey assesses the mental health literacy of teachers as it relates to students and within the classroom context. Data analyses were run to explore the internal reliability, discriminant validity, and feasibility of the survey. Analyses indicate moderate to high reliability (Cronbach's alpha=.743), providing psychometric support for the use of the TSMHLS with pre-service teachers. Pre-service teachers' discrimination between clinical symptomology and typical development was significant, providing further support for the survey's use in understanding pre-service teachers' knowledge of SEB concerns. Finally, feasibility was assessed through average completion time for the online survey (24 minutes), and results indicate that the survey could be feasibly implemented during pre-service teacher training. These findings lend support to the TSMHLS as an appropriate measure to evaluate pre-service teachers' knowledge of student mental health.
\end{abstract}

Keywords: mental health, school, teachers, students

\section{Introduction}

One in five children will experience symptoms of a social, emotional or behavioral (SEB) disorder before the time they turn 18 (Merikangas et al, 2010). These symptoms can manifest at schools in teachers' classrooms and put students at a greater risk for interpersonal conflicts, poor academic achievement, lower school attendance, entrance into special education programs, school failure, and potential engagement in risky behaviors, such as suicide (Aseltine, Gore, \& Gordon, 2000; Bradshaw et al., 2008; Davis, Kreczek, \& Mcintosh, 2006; Hawton, Saunders, \& O’Connor, 2012; McWhirter \& Page, 1999; Wagner,1995). Given these implications, there is a need for prevention and early intervention for students displaying SEB concern in order to prevent the development of more serious concerns. 


\section{Teacher Referral and Student Access to Resources}

Early identification and intervention are important for preventing long-term deleterious outcomes for youth struggling with mental health disorders. The majority of those youths identified and receiving interventions for SEB concerns do so within schools, with teachers being instrumental to identifying students needing intervention (Rones \& Hoagwood, 2000). Teachers' ability to identify problems is critical in students' access to services. Prior research has indicated providers', including teachers', perception of SEB problems and severity are stronger predictors of students' access to intervention than student self-reports of their SEB functioning (Stiffman et al., 2000). Although teachers' perceptions are strong predictors, prior research has also shown that teachers have difficulty identifying students with internalizing behaviors (Cunningham \& Suldo, 2014; Neil \& Smith, 2017), especially when compared to their concern for students with externalizing behavior problems (Splett et al., 2019). Internalizing behaviors may include symptoms of disorders such as anxiety or depression, which tend to show less obvious symptoms in the classroom. Teachers also report being more concerned about and more likely to refer students showing externalizing behaviors than those exhibiting internalizing behaviors (Chang \& Sue, 2003; Loades \& Mastroyannopoulou, 2010). Externalizing behaviors present overt symptoms such as classroom disruption or violation of school policies as a result of an externalizing disorder. The need for teacher training to recognize mental health concerns is especially important for students exhibiting internalizing behaviors because, as discussed next, they tend to go unidentified due to the absence of obvious symptoms.

\section{Teacher Training and Knowledge on Student Mental Health}

Teachers and other staff remain crucial participants in the cycle of identifying and referring students for services in response to SEB concerns. However, teachers report inadequate training and knowledge to identify students' SEB needs in schools (Reinke, Stormont, Herman, Puri, \& Goel, 2011). Thus, there is a need to develop effective training programs for teachers regarding identification of SEB concerns (Reinke et al., 2011) especially given that at least $25 \%$ of students exhibiting mental health concerns do not receive necessary treatment and the gap is greater for students within internalizing behavior concerns than those with externalizing (Splett et al., 2018). This problem of students not receiving services can be due to both the teachers' identification of the behavior and level of concern regarding the behavior, as discussed by Loades and Mastroyannopoulou (2010) who found teachers were more concerned with externalizing 
behavior vignettes than internalizing. Therefore, in order to develop and evaluate teacher training for SEB concerns, measures of teachers' knowledge, identification, and concern are needed.

\section{Measures of Teacher Mental Health Literacy}

Unfortunately, current measures of teacher knowledge of student SEB concerns do not address all areas of concern (Splett et al., 2019). For example, current measures narrowly assess teachers' identification of specific and less common disorders (e.g., Specific Phobia) and/or do not cover the continuum of SEB concerns (e.g., SEB strengths or comorbid problems). Thus, this proposal aims to address the gap in the research by examining the psychometric properties and feasibility of a measure developed to assess teachers' identification of comorbid SEB profiles, including prosocial, normative, at-risk, and extremely elevated levels.

Specifically, the study provides psychometric examination of the Teacher School Mental Health Literacy Survey (TSMHLS; Brann, Bidwell, Boone, Dinnen, \& Splett, 2018). To assess psychometric properties of scores on the TSMHLS and feasibility, we aimed to answer the following questions regarding the TSMHLS “(1) To what extent are subscale scores of the TSMHL survey reliable with a pre-service teacher population?, (2) How well can pre-service teachers discriminate between the intended SEB profiles?, and (3) How feasible is the survey to administer to teachers?" Given that the TSMHLS was developed by experts and its content was validated by researchers and practitioners in the field, we hypothesize scores on the measure will be valid and reliable in the current study. In sum, the current study assists in determining the psychometric properties of scores on the TSMHLS and feasibility within a population of preservice teachers.

\section{Methods}

\section{Participants and Settings}

Participants in this cross-sectional study were recruited from the undergraduate and graduate students enrolled in the Unified Elementary $(n=240)$ and Early Childhood $(n=8)$ Education programs at the University of Florida, who will be referred to as pre-service teachers for the remainder of the paper. This represents a convenience sample as pre-service teachers at the University of Florida were chosen based on feasibility of study implementation. A total of 38 pre-service teachers in the two education programs participated. A majority of the sample identified as Caucasian (84\%) and female (100\%). The participants' years of training within the 
program ranged from first semester $(23 \%)$ to sixth semester $(6 \%)$ pre-service teachers, with most of the participants $(40 \%)$ in their third semester of study. See Table 1 for demographics.

\begin{tabular}{|c|c|c|}
\hline \multicolumn{3}{|l|}{ Sex } \\
\hline & Female & $100 \%$ \\
\hline & Male & $0 \%$ \\
\hline \multicolumn{3}{|l|}{ Race } \\
\hline & Caucasian & $84 \%$ \\
\hline & Black or African American & $8 \%$ \\
\hline & Asian or Asian American & $8 \%$ \\
\hline \multicolumn{3}{|c|}{ Hispanic } \\
\hline & Yes & $21 \%$ \\
\hline & No & $79 \%$ \\
\hline \multicolumn{3}{|c|}{ Classification } \\
\hline & Junior (1-2 semesters) & $31 \%$ \\
\hline & Senior (3-4 semesters) & $47 \%$ \\
\hline & Graduate student ( $5-6$ semesters) & $22 \%$ \\
\hline
\end{tabular}

\section{Procedures}

Following IRB approval, the survey was administered to the participating pre-service teachers via a Qualtrics survey. The survey was voluntary within the targeted sample and 43 responses were collected in total, with 38 responses completing all the survey questions. The online survey was created based on existing measures, composed of closed and open-ended questions intended to assess teachers' SEB knowledge, help-seeking efficacy, and beliefs. It also includes vignettes describing hypothetical students' SEB profiles, which assess teachers' SEB identification in an applied context. Pre-service teachers were emailed a link to the online survey and completed the survey via the online portal. After the initial email was sent, the study facilitator spoke to those who were eligible to participate during a regularly scheduled class and briefly explained the project, inviting participation through the emailed survey. The survey was emailed out a second time after these brief presentations. As an incentive for participating, those who completed the survey and submitted their name were entered into a lottery drawing to win a $\$ 50$ gift card to a popular online retailor. The data were exported from the online platform and coded by an advanced undergraduate research assistant.

Measures teacher survey. The Teacher School Mental Health Literacy Survey was developed to assess teachers' SEB knowledge, attitudes, self-efficacy, and beliefs regarding SEB concerns, teachers' roles and help-seeking. The survey consisted of 84 items with two different formats, Likert scales and multiple-choice questions. The survey included ten background and 
demographic questions; 66 items assessing teachers' attitudes, beliefs, self-efficacy and knowledge around SEB concerns; and 18 vignette-based items measuring teacher ability to identify common SEB needs (Brann et al., 2018). The 18 vignette-based items were answered in regards to three vignettes describing a child in elementary school with either (a) common externalizing behavior problems (i.e., arguing, yelling, pushing), (b) internalizing behavior problems (i.e., withdrawn from peers, nervousness), or (c) developmentally-typical SEB functioning. Each vignette was followed by six questions, querying respondents' recognition of the problem, severity of the problem, endorsement of the child's need for help, self-rating of ability and knowledge to assist the child with a problem, and knowledge of referrals for the student of concern.

\section{Data Analyses}

We carried out all analyses using SPSS (IBM Corp. Released 2017. IBM SPSS Statistics for Windows, Version 25.0. Armonk, NY: IBM Corp). For the first research questions, we calculated internal consistency for each subscale using Cronbach's alpha to determine the reliability of each scale. Alpha values can be classified as minimally acceptable (0.65), acceptable (0.70) and optimal (0.80) (DeVellis, 1991; Nunnally \& Bernstein, 1994). To answer the second research question, we ran a series of paired-sample t-test comparing the mean scores of participants' ratings of problem recognition following each vignette in order to determine if pre-service teachers could discriminate between clinically significant vignettes and a typically developing vignette. Finally, feasibility of survey use was assessed using the average completion time of the complete responses to the online survey.

\section{Results}

\section{Internal Reliability}

For each subscale, Cronbach's alpha was used to determine internal reliability. For the attitudes scale, which consisted of 10 items reflecting teachers' beliefs about teacher involvement with mental health promotion and intervention in schools, internal reliability was high $(\alpha=.882)$. For the beliefs about mental health scale, which consisted of eight items concerning teachers' beliefs about mental health conditions, internal reliability was low $(\alpha=.344)$. The knowledge scale consisted of 12 items assessing teachers' knowledge regarding warning signs for the development of mental health concern, and internal consistency was high 
$(\alpha=.897)$. The knowledge of best practices scale, which consisted of 14 questions pertaining to teacher skills and practices regarding mental and social emotional concern, had a medium to high internal reliability ( $\alpha=$.708). Teacher self-efficacy scale, measured by 15 -items regarding teachers' perception of their own abilities to recognize and address mental health concerns, had a high internal consistency $(\alpha=.936)$. The teacher belief about help scale, which consisted of 5items pertaining to teacher understanding and beliefs about receiving help in school for mental health concerns, had a moderate internal consistency of $(\alpha=.693)$. However, one question on this scale was dropped from analysis because it pertained to understanding specific school-level procedures, and many pre-service teacher participants did not work full time in a school setting. With the discrepant question removed, the internal consistency of the remaining four items was high $(\alpha=.906)$.

\section{Discriminant Validity}

Pre-service teachers' ability to discriminate between clinical symptomology and typical development was assessed using a series of paired-sample t-tests between the ratings of problem recognition following each of the three different vignettes. Responses to the problem recognition question assessing teachers' agreement that the vignette description presented a mental health concern were compared. The question was rated on a six-point Likert scale from Strongly Disagree to Strongly Agree that the vignette depicted a mental health concern. There was a statistically significant mean difference $(t=13.855, p<.01)$ between teacher rating of a mental health concern for the internalizing vignette $(M=4.94, S D=.854)$ and the typically developing vignette $(M=$ $2.31, S D=1.078)$. There was also a statistically significant mean difference $(t=11.122, p, .01)$ between teacher rating of a mental health concern for the externalizing vignette $(M=4.38, S D$ $=1.025)$ and the typically developing vignette $(M=2.31, S D=1.078)$.

\section{Feasibility}

Feasibility of survey use was assessed using the completion time of the 38 complete responses to the online survey. The online survey platform, Qualtrics, recorded completion time for each survey. The average completion time for the 114 survey items was 24.49 minutes, with a minimal completion time of 8.57 minutes and a maximum completion time of 89.42 minutes. 


\section{Discussion}

The goal of the study was to validate an existing measure of teachers' knowledge and perceptions of student mental health to guide future use of such measures in the development and testing of teacher training efforts in this area. It was hypothesized that such a measure would yield valid and reliable scores and begin filling the gap of teachers reporting inadequate training. Overall, the study supported our hypotheses showing that the TSMHLS yields internally consistent scores with adequate discriminant validity between problem severity and a feasible completion time. Internal consistency analysis indicates medium to high internal consistency for five out of the six subscales on the survey, and items measured in each of those subscales show low variability. This consistency is desirable when measuring knowledge on a certain subject. For the beliefs about mental health scale, the internal reliability $(\alpha=.344)$ for this scale could be explained by a few reasons. The questions ask about various factors within mental health including teacher's role, the cause of student mental health concerns, and outside factors including the family, the classroom, and general health. The responses within this scale may have more variability based on individual belief systems, and further analyses looking at patterns of responses are necessary to understand how different groups answered the items on this scale. When utilizing these questions in the future it may be beneficial to remove the few questions that skewed the reliability score in order to improve greater consistency, however it should be noted that our small sample size was also limited in demographic variability, so a larger and more diverse pool of individuals may provide greater insight.

The discriminant validity tests showed statistically significant differences in how participants rated the mental health concern of vignettes that displayed clinical problem behaviors versus typical behavior. The statistical significance indicates that pre-service teachers can differentiate between different levels of behavior problems severity. Overall, the level of concern was much lower for typical problems and much higher for clinical problems. The sample of pre-service teachers surveyed were able to discriminate between typical and concerning behaviors, even though their knowledge of student mental health would be expected to be lower, due to less experience in the classroom. This provides further support that the vignettes are demonstrating different levels of behaviors as intended. It also raises additional future research questions. Is there greater exposure to and education toward mental health concerns in current pre-service teacher training than previously? If so, does this knowledge render more successful teacher referral of 
students or is there a lack of applied knowledge? Overall, the results are meaningful for further exploration of the teacher's role in student mental health.

Feasibility of implementing the survey was measured through the number of minutes of completion time from opening the survey to submitting it. The average survey time for this study, at about 24 minutes, was greater than the previously measured completion time of approximately 10 minutes (Splett et al, 2019). The reasoning could be because the survey was administered online and could be completed over an unrestricted period of time, whereas the previous version of this survey was completed on paper during a specific time period. The survey link online could have been opened on a computer and placed in the background, providing more convenience to participants, yet also calculating minutes that were not technically used in survey completion. Knowing this, an additional question could be placed at the end asking the participant to estimate the number of minutes actually used to complete the survey to gain better insight into feasibility.

\section{Limitations}

This study provided additional support for the use of the TSMHLS by examining its psychometric properties in a new sample of pre-service teachers in educator preparation programs where training in children's mental health should ideally begin. Despite the importance of this study, interpretation of findings should only be made in light of several limitations. Reliability may be limited because this survey was utilized within a sample of participants with the same or very similar pre-service teacher training. Therefore, the results of this study may not be able to extend to the general population of in-service or pre-service teachers. Additionally, this study only extended to those pre-service teachers who are training to be early childhood or elementary educators, many of who are not planning to teach in the middle-school or high school grades. This is important to note because prior research has shown that the referral rate for mental health concerns is much lower among elementary teachers than high school teachers (Green et al., 2017). As for discriminant validity, this study may also have limitations because it collected from a sample size that is smaller than anticipated and desired. Additionally, the sample had $100 \%$ female participants. Although this is consistent with the gender composition of the teaching profession, which is still predominately female at the elementary and secondary level, the lack of any male participation may bias results. Finally, feasibility of this study is limited because it was not implemented within a school or with in-service teachers. Instead, it was implemented with preservice teachers who may have more time to be able to complete the survey, therefore this 
measurement may be unreliable for the intended general population of in-service teachers. The survey could also be opened and completed in more than one sitting, so our data on feasibility may not accurately depict the average time required to complete survey from start to finish.

\section{Conclusion}

This study, although presenting limitations, brings light to the topic of mental health in schools and the role of the teacher in student referral. Teachers do not receive adequate pre-service teacher training on recognizing mental health concerns or knowledge of their role in this. More mental health conversations and content during pre-service teacher programs are needed in order to shift away from the perceptions some teachers have of student mental health being beyond their expertise or role responsibilities. Considering mental health in schools is a topic of concern, teachers need the support to recognize and embrace their role in this. If more education and experience with student mental health is implemented at the pre-service teacher level, our schools will begin to be filled with more teachers who have knowledge of and feel more prepared to exercise their role in student referral. Hopefully this will lead to more students receiving the interventions needed to support and guide their mental health and well being in schools.

\section{Implications}

Comprehensive and validated tools, such as a survey, used to measure teachers' knowledge of student mental health concerns, serve as the initial step to having more knowledgeable and prepared teachers to refer students to services. Schools should use measures, such as the TSMHLS, to evaluate and then guide their professional development and training for teachers on this topic. To educate pre-service teachers before they enter the field, colleges or universities can also use a measure like the TSMHLS to understand the topics that should be included in pre-service teacher courses or to guide the implementation of a new microcourse on student mental health. With teachers who have greater knowledge and self-efficacy for identifying students of mental health concern, the system of services in schools can improve through successfully intervening preventative services with students. Overall, teachers should be exposed to the topic of school mental health and be made aware of their invaluable role in student access to services in order to continue mending the gap. 


\section{Acknowledgements}

A special thank you to Dr. Joni Splett for her guidance and mentorship. Additional gratitude to Kathryn M.Trainor and Lia K.Thibodaux for their assistance in supporting and developing this research project.

\section{References}

Aseltine Jr, R. H., Gore, S., \& Gordon, J. (2000). Life stress, anger and anxiety, and delinquency: An empirical test of general strain theory. Journal of Health and Social Behavior, 256-275. doi: $10.2307 / 2676320$

Brann, K., Bidwell, S., Boone, W., Dinnen, H., \& Splett, J. (2018). Teacher School Mental Health Literacy Survey [unpublished instrument].

Chang, D. F., \& Sue, S. (2003). The effects of race and problem type on teachers' assessments of student behavior. Journal of Consulting and Clinical Psychology, 71(2), 235-242. doi:10.1037/0022006X.71.2.235

Cunningham, J. M., \& Suldo, S. M. (2014). Accuracy of teachers in identifying elementary school students who report at-risk levels of anxiety and depression. School Mental Health, 6(4), 237250. doi: 10.1007/s12310-014-9125-9

Davis, A. S., Kruczek, T., \& Mcintosh, D. E. (2006). Understanding and treating psychopathology in schools: Introduction to the special issue. Psychology in the Schools, 43, 413-417. doi: 10.1002/pits.20155

Green, J. G., Guzman, J., Didaskalou, E., Harbaugh, A. G., Segal, N., \& LaBillois, J. (2018). Teacher identification of student emotional and behavioral problems and provision of early supporters: A vignette-based study. Journal of Emotional and Behavioral Disorders, 26(4), 225-242. doi: $10.1177 / 1063426617740879$

Loades, M. E., \& Mastroyannopoulou, K. (2010). Teachers' recognition of children's mental health problems. Child and Adolescent Mental Health, 15(3), 150-156. doi: 10.1111/j.14753588.2009.00551.x

Merikangas, K.R., He, J., Burstein, M., Swanson, S.A., Avenevoli, S., Cui, L., Benjet, C., Georgiades, K., \& Swendsen, J. (2010). Lifetime prevalence of mental disorders in U.S. adolescents: Results from the national comorbidity study-adolescent supplement (NCS-A). Journal of the American Academy of Child and Adolescent Psychiatry, 49(10), doi: 980-989. 10.1016/jaac.2010.05.017

Reinke, W. M., Stormont, M., Herman, K. C., Puri, R., \& Goel, N. (2011). Supporting children's mental health in schools: Teacher perceptions of needs, roles, and barriers. School Psychology Quarterly, 26(1), 1-13. doi:10.1037/a0022714

Rones, M., \& Hoagwood, K. (2000). School-based mental health services: A research review. Clinical Child and Family Psychology Review, 3(4), 223-241. doi:10.1023/A:1026425104386 
Splett, J. W., Gibson, N., Garzona, M., Wojtalewicz, D., Raborn, A., \& Reinke, W. M. (2019). Teacher recognition, concern and referral of internalizing behavior problems. School Mental Health,1-12. doi: 10.1007/s12310-018-09303-z

Splett, J. W., George, M. W., Zaheer, I., Weist, M.D., Evans, S. W., \& Kern, L. (2018). Symptom profiles and mental health services received among referred adolescents. School Mental Health, 10(2), 96110. doi:10.1007/s12310-017-9244-1

Stiffman, A. R., Hadley-Ives, E., Doré, P., Polgar, M., Horvath, V. E., Striley, C., \& Elze, D. (2000). Youths' access to mental health services: The role of providers' training, resource connectivity and assessment of need. Mental Health Services Research, 2(3), 141-154. doi:10.1023/A:1010189710028

Wagner, M. (1995). Outcomes for youths with serious emotional disturbance in secondary school and early adulthood. The Future of Children, 5, 90-112. 